\title{
High-Resolution Dynamic Cardiac MRI on Small Animals using Reconstruction based on Split Bregman Methodology
}

\author{
P. Montesinos, J. F. Pérez-Juste Abascal, J. Chamorro, C. Chavarrías, M. Benito, J.J. Vaquero, Member, IEEE, and M. Desco
}

\begin{abstract}
Dynamic cardiac magnetic resonance imaging in small animals is an important tool in the study of cardiovascular diseases. The reduction of the long acquisition times required for cardiovascular applications is crucial to achieve good spatiotemporal resolution and signal-to-noise ratio. Nowadays there are many acceleration techniques which can reduce acquisition time, including compressed sensing technique. Compressed sensing allows image reconstruction from undersampled data, by means of a non linear reconstruction which minimizes the total variation of the image. The recently appeared Split Bregman methodology has proved to be more computationally efficient to solve this problem than classic optimization methods. In the case of dynamic magnetic resonance imaging, compressed sensing can exploit time sparsity by the minimization of total variation across both space and time. In this work, we propose and validate the Split Bregman method to minimize spatial and time total variation, and apply this method to accelerate cardiac cine acquisitions in rats. We found that applying a quasi-random variable density pattern along the phaseencoding direction, accelerations up to a factor 5 are possible with low error. In the future, we expect to obtain higher accelerations using spatiotemporal undersampling.
\end{abstract}

\section{INTRODUCTION}

A CQUISITION time required to obtain images with $A$ good spatiotemporal resolution and signal-to-noise ratio in cardiovascular applications is prohibitively long.

This work is supported in part by Ministerio de Ciencia e Innovación (Red RECA VA), Comunidad de Madrid and Fondos FEDER (ARTEMIS project S2009DPI 1802).

P. Montesinos is with the Departamento de Bioingeniería e Ingeniería Aeroespacial, Universidad Carlos III de Madrid, Spain (e mail: pmontesinos@hggm.es).

J. F. Pérez Juste Abascal is with the Departamento de Bioingeniería e Ingeniería Aeroespacial, Universidad Carlos III de Madrid, Spain (e mail: jabascal@hggm.es).

J. Chamorro is with the Departamento de Bioingeniería e Ingeniería Aeroespacial, Universidad Carlos III de Madrid, Spain (e mail: jchamorro@hggm.es).

C. Chavarrias is with the Unidad de Medicina y Cirugía Experimental, Hospital General Universitario Gregorio Marañón, Madrid, Spain (e mail: cchavarrias@hggm.es).

M. Benito is with the Unidad de Medicina y Cirugía Experimental, Hospital General Universitario Gregorio Maranón, Madrid, Spain (e mail: mbenito@hggm.es).

J. J. Vaquero is with the Departamento de Bioingeniería e Ingeniería Aeroespacial, Universidad Carlos III de Madrid, Spain (e mail: juanjose.vaquero@uc3m.es).

M. Desco is with the Unidad de Medicina y Cirugía Experimental, Hospital General Universitario Gregorio Marañón, Madrid, Spain and the Departamento de Bioingeniería e Ingeniería Aeroespacial, Universidad Carlos III de Madrid, Spain (e mail: manuel.desco@uc3m.es).
Thus there is a great interest in acceleration techniques, which has promoted the apparition of a great amount of new accelerating methods in the last few years, including compressed sensing techniques [1]-[7].

Compressed sensing allows exact reconstruction from randomly undersampled data by a suitable nonlinear approach, with the only assumption that the image is sparse in the pixel representation or in a certain transform domain [8]. The quality of the reconstruction depends on two issues: the type of data undersampling and the degree of sparsity of the image in a certain transform domain [9]-[11].

While only some images are directly sparse in the image domain, as is the case of angiography, most of them are sparse in the gradient domain. To make an image sparse in this domain, it is necessary to minimize the L1 norm of the gradient of the image, what is call total variation (TV). In the particular case of dynamic images, compressed sensing can exploit sparsity by the minimization of total variation in space and time.

Among the great variety of existent reconstruction algorithms, Split Bregman method [12], [13] and similar methods [14] solve the constrained optimization problem in a more efficient computationally way than other classic optimization methods.

In this work we extend the Split Bregman formulation to the minimization of the total variation in both space and time and we study the feasibility of applying this method to cardiac cine studies on rats. In order to achieve this, several simulations were performed over a complete cardiac cine dataset acquired with retrospective gating [16]. This cine technique is very useful to assess cardiac function, to evaluate heart motility and to quantify the ejection fraction in small animals.

\section{METHODS}

\section{A. Image Reconstruction}

The image reconstruction problem is solved based on a constrained optimization approach described by [8]

$$
\min _{u}\|u\|_{1}+\|\nabla u\|_{1}+\left\|D_{\imath} u\right\|_{1} \text { such that } F u=f,
$$

where $\nabla=\left(D_{x}, D_{y}\right)$ is the gradient, and $D_{x}, D_{y}$ and $D_{t}$ are the spatial and time derivatives respectively, and \|\|$_{1}$ is the L1 norm, $\|\nabla u\|_{1}$ is the spatial total variation, and $\left\|D_{t} u\right\|_{1}$ is the time total variation. 
This can be easy and efficiently solved using the Split Bregman formulation that defines an equivalent problem

$\min _{d_{x}, d_{y}, d_{t}, u}\left\|\left(d_{x}, d_{y}\right)\right\|_{1}+\left\|d_{t}\right\|_{1}+\frac{\mu}{2}\left\|F u-f^{k}\right\|_{2}^{2}$

$+\frac{\lambda}{2}\left\|d_{x}-D_{x} u-b_{x}^{k}\right\|_{2}^{2}+\frac{\lambda}{2}\left\|d_{y}-D_{y} u-b_{y}^{k}\right\|_{2}^{2}$

$+\frac{\lambda}{2}\left\|d_{t}-D_{t} u-b_{t}^{k}\right\|_{2}^{2}$

where each $b_{i}$ represents the Bregman iteration that imposes a constraint, and the new variables, $d_{i}$, allow decoupling L2 and L1 functionals $[13,17]$.

\section{B. Data Sets}

Cardiac cine of a healthy rat was acquired using a 7T Bruker Biospec 70/20 scanner with a linear coil resonator for transmission and a dedicated cardiac phased array coil for reception.

The original fully sampled cartesian data were acquired with an IntraGate FLASH sequence [16] with the following parameters: $\mathrm{TE}=2.43 \mathrm{~ms}$, TR $=8 \mathrm{~ms}$, number of total repetitions $=200$, number of frames $=8$, matrix size $=$ $192 \times 192, \mathrm{FOV}=4.8 \mathrm{~cm} \times 4.8 \mathrm{~cm}$, slice thickness $=1.2 \mathrm{~mm}$ with a total acquisition time of $5 \mathrm{~min} 7 \mathrm{~s}$.

All the reconstructions were performed separately for each element of the phased array, and then combined with a sum of squares.

\section{Sampling Patterns}

For this application, acquisition time depends directly on the number of acquired phase-encoding lines, so undersampled data were obtained by quasi-randomly removing phase-encoding lines from the original data.

The undersampling patterns were created based on a variable probability density function which maintains a fix percentage of data around the center of the k-space and gives more probability of being preserve to lines according to its distance to the k-space center, where is stored most part of the image information (Fig. 1).

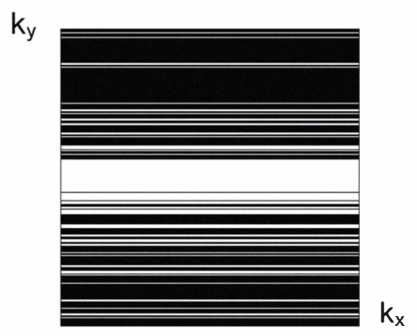

Fig. 1. Undersampling pattern generated from a probability density function. Black lines represent eliminated phased encoding lines from the original complete data.

To validate the proposed method and to determine the highest acceleration factor achievable for this application we performed reconstructions of undersampled data maintaining from $10 \%$ up to $40 \%$ of the original data in $5 \%$ steps.

\section{Evaluation of the reconstructed images}

Besides visual inspection of reconstructed images, mean squared error (MSE) was calculated over a defined region of interest around the heart.

\section{RESULTS}

In Fig. 2 mean squared error obtained for the different cine frames as a function of the percentage of preserved data is shown.

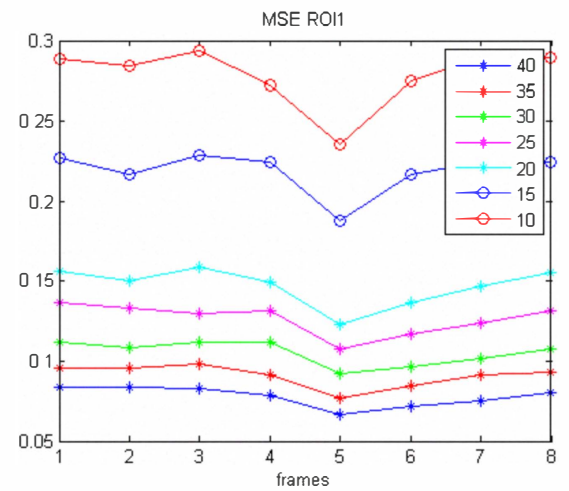

Fig. 2. Mean squared error for each frame as a function of the quantity of preserved data.

In Fig. 3 different reconstructed images are compared. A) Fully sampled data reconstructed by Fourier transform. B) Undersampled data $(20 \%$ of the original data $)$, reconstructed with the proposed Split Bregman method. C) Undersampled data $(35 \%$ of the original data), reconstructed with Split Bregman. D) Undersampled data ( $35 \%$ of the original data), reconstructed by zero filling and Fourier transform.
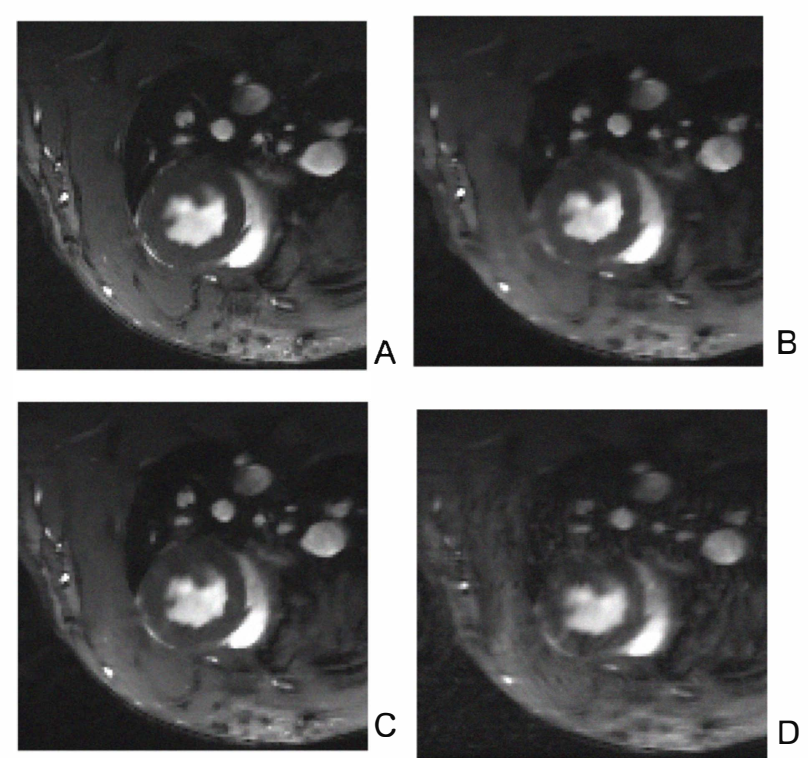

Fig. 3. A) Fully sampled image. B) Split Bregman reconstruction of undersampled data $(20 \%)$. C) Split Bregman reconstruction of undersampled data (35\%). D) Zero filling reconstruction of undersampled data $(35 \%)$. 


\section{DISCUSSION}

In this work we propose the Split Bregman methodology for the minimization of total variation in both space and time for dynamic images. This methodology allows reconstructing images from only a $20 \%$ of the original data with low error.

In Fig. 3 the quality of the image reconstructed with a $35 \%$ of the original data $(\mathrm{C})$ is similar to the original one (A). If we use only a $20 \%$ of the original data, the reconstructed image (B) has a quite reasonable quality, although it has lower contrast and some artifacts.

For the same amount of preserved data the proposed methodology is conspicuously better than a reconstruction based on zero filling and Fourier transform (C-D).

If we take into account both the visual inspection of the images and the mean squared error values obtained, we can achieve accelerations superiors to a 2.8 factor $(35 \%$ of the original data). This factor can be increased up to 5 (20\% of the original data) depending on the concrete application we are working with and on tolerance to artifacts desired in the final image.

This acceleration so far is higher than what we could expect with a conventional parallel imaging method (acceleration factor 2).

Although in these preliminary results the same sampling pattern was applied to all the cine frames, we expect to improve these results by applying more complex undersampling patterns, randomized in space and time.

In conclusion, the proposed Split Bregman formulation for the minimization of the time and spatial TV seems a promising method to accelerate dynamic cardiac MRI in small animals.

\section{REFERENCES}

[1] B. Madore et al., "Unaliasing by Fourier encoding the overlaps using the temporal dimension (UNFOLD), applied to cardiac imaging and AMRI," Magn Reson Med, vol. 42, pp. 813 828, 1999.
[2] J. Tsao et al., "k t BLAST and $k \mathrm{t}$ SENSE: dynamic MRI with high frame rate exploiting spatiotemporal correlations," Magn Reson Med, vol. 50, pp. 1031 1042, 2003

[3] M. Lustig et al., "k t SPARSE: High Frame Rate Dynamic MRI Exploiting Spatio Temporal Sparsity" Proc. Int. Soc. Mag. Reson. Med., Seattle, WA, 2006, p. 2420

[4] N. Aggarwal et al, "Patient adapted reconstruction and acquisition dynamic imaging method (PARADIGM) for MRI," Inverse Probl, vol. 24,2008

[5] H. Jung et al., "k t FOCUSS: a general compressed sensing framework for high resolution dynamic MRI," Magn Reson Med, vol. 61, pp. 103 116, 2009.

[6] H. Pedersen et al., " $\mathrm{k}$ PCA: temporally constrained $\mathrm{k} t$ BLAST reconstruction using principal component análisis". Magn Reson Med. 2009 Sep;62(3):706 16.

[7] D. Liang et al., "K-T ISD: Compressed sensing with iterative support detection for dynamic MRI", to appear Proc. of IEEE International Symposium on Biomedical Imaging (ISBI), 2011. accepted, 10/2010.

[8] E. J. Candès et al., "Robust uncertainty principles: exact signal reconstruction from highly incomplete frequency information". IEEE Trans. Inform. Theory, 52(2) 489 509. 2006.

[9] M. Lustig et al., "Sparse MRI: The Application of Compressed Sensing for Rapid MR Imaging", Magnetic Resonance in Medicine, 2007; 58(6):1182 1195

[10] A.M. Bruckstein et al., "From Sparse Solutions of Systems of Equations to Sparse Modeling of Signals and Images", SIAM Review, Vol. 51, No. 1, Pages 34 81, February 2009.

[11] E. Garduño et al., "Reconstruction from a few projections by 11 minimization of the Haar transform", Inverse Problems 27, 055006, 2011

[12] S. Osher et al., "An Iterative Regularization Method for Total Variation Based Image Restoration". Multiscale Model. Simul. 4, pp. $460489,2005$.

[13] T. Goldstein et al., "The Split Bregman Method for L1 Regularized Problems." SIAM Journal on Imaging Sciences. Volume 2, Issue 2, pp. 323343 (2009).

[14] L. He et al. "MR image reconstruction from undersampled data by using the iterative refinement procedure". PAMM, 7: 1011207 1011208 .

[15] S. Lingala et al., "Accelerated Dynamic MRI Exploiting Sparsity and Low Rank Structure: k t SLR", IEEE Transactions on Medical Imaging, 30, Issue 5, 1042 1054, 2011.

[16] S.M Bovens et al., "Evaluation of infarcted murine heart function: comparison of prospectively triggered with self gated MRI" NMR Biomed. 2011 Apr;24(3):307 15.

[17] Y. Wang et al., "A new alternating minimization algorithm for total variation image reconstruction", SIAM J. Imaging Sci, 248 272, 2008 